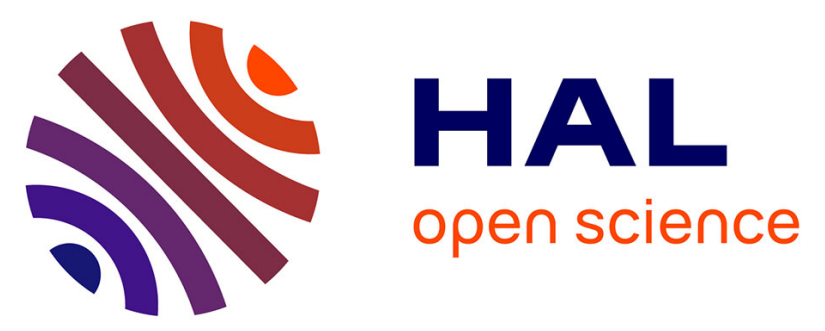

\title{
Comment on "Density functional theory analysis of structural and electronic properties of orthorhombic perovskite CH3NH3PbI3" by Y. Wang et al., Phys. Chem. Chem. Phys., 2014, 16, 1424-1429
}

\author{
Jacky Even, Laurent Pedesseau, Claudine Katan
}

\section{To cite this version:}

Jacky Even, Laurent Pedesseau, Claudine Katan. Comment on "Density functional theory analysis of structural and electronic properties of orthorhombic perovskite CH3NH3PbI3" by Y. Wang et al., Phys. Chem. Chem. Phys., 2014, 16, 1424-1429. Physical Chemistry Chemical Physics, 2014, 16 (16), pp.8697-8698. 10.1039/C3CP55006K . hal-01077152

\section{HAL Id: hal-01077152 \\ https://hal.science/hal-01077152}

Submitted on 25 Nov 2016

HAL is a multi-disciplinary open access archive for the deposit and dissemination of scientific research documents, whether they are published or not. The documents may come from teaching and research institutions in France or abroad, or from public or private research centers.
L'archive ouverte pluridisciplinaire HAL, est destinée au dépôt et à la diffusion de documents scientifiques de niveau recherche, publiés ou non, émanant des établissements d'enseignement et de recherche français ou étrangers, des laboratoires publics ou privés. 


\title{
Comment on "Density functional theory analysis of structural and electronic properties of orthorhombic perovskite CH3NH3PbI3" by Y. Wang et al., Phys. Chem. Chem. Phys., 2014, 16, 1424-1429
}

\author{
J. Even ${ }^{*}$, L. Pedesseau a and C. Katan ${ }^{b}$ \\ aUniversité Européenne de Bretagne INSA, FOTON UMR 6082, 35708 Rennes, France. \\ E-mail: jacky.even@insa-rennes.fr \\ ${ }^{b}$ CNRS, Institut des Sciences Chimiques de Rennes, UMR 6226, 35042 Rennes, France
}

Yun Wang et al. used density functional theory (DFT) to investigate the orthorhombic phase of $\mathrm{CH}_{3} \mathrm{NH}_{3} \mathrm{PbI}_{3}$, which has recently shown outstanding properties for photovoltaic applications. Whereas their analysis of ground state properties may represent a valuable contribution to understanding this class of materials, effects of spin-orbit coupling (SOC) cannot be overlooked as was shown in earlier studies. Moreover, their discussion on optical properties may be misleading for non-DFT-experts, and the nice agreement between experimental and calculated band gap is fortuitous, stemming from error cancellations between SOC and many-body effects. Lastly, Bader charges suggest potential problems during crystal structure optimization.

The recent paper by Yun Wang et al. reports results based on density functional theory (DFT) simulations aiming to investigate structural, electronic and optical properties of the low temperature orthorhombic phase of $\mathrm{CH}_{3} \mathrm{NH}_{3} \mathrm{PbI}_{3}$. This compound and related mixed-halide hybrid perovskites have attracted considerable research efforts in the past twelve months, as they are expected to offer a promising route towards low-cost and efficient solar cells. ${ }^{1,2}$

While their structural study, revealing the importance to account properly for van der Waals interactions, may be a valuable result (vide infra), most of the discussion is skewed as spin-orbit coupling (SOC) and has been completely disregarded. This is quite surprising for a lead-based material and given available papers in the literature evidencing the importance of SOC effects in these materials. ${ }^{3,4}$ Moreover, statements such as "suitable method for this material" in addition to the detailed discussion on optical properties may lead non-DFT-experts to wrong conclusions for the assessment of physical properties and methods and/or future theoretical work.

In fact, earlier DFT calculations performed both for $2 \mathrm{D}^{3}$ and $3 \mathrm{D}^{4}$ lead-based hybrid and allinorganic perovskites ${ }^{4}$ evidenced the dramatic effect of SOC on their optical properties, showing that their conduction bands undergo a giant spin-orbit split off. Moreover, in ref. 4, the very same low temperature orthorhombic phase of $\mathrm{CH}_{3} \mathrm{NH}_{3} \mathrm{PbI}_{3}$ was explicitly considered with detailed discussion on the importance of SOC on the strength and polarisation properties of the absorption of $\mathrm{CH}_{3} \mathrm{NH}_{3} \mathrm{PbI}_{3}$. These results showed that while SOC can often be considered as a small correction to standard DFT calculations, and thus be neglected, this is not the case for such 
hybrid perovskites. The conduction band state at the band gap, of the high, room and low temperature phases, is always of spin-orbit split off type..$^{4-6}$ This has a direct consequence on the band gap absorption that is roughly overestimated by a factor of three in DFT calculations without SOC, as compared to calculations with SOC, for lead-based perovskites. Proper account of SOC is also mandatory to understand the polarization properties.,

Besides, the intermediate room temperature tetragonal phase of $\mathrm{CH}_{3} \mathrm{NH}_{3} \mathrm{PbI}_{3}$ is the most important for applications. ${ }^{4,5}$ The tetragonal space group is only briefly mentioned by Yun Wang et al. ("I $\mathrm{I} / \mathrm{m}$ "). A tetragonal centrosymmetric phase, with the $\mathrm{I} 4 / \mathrm{mcm}$ space group, was indeed proposed in the literature. ${ }^{7,8}$ More recently, a new high temperature phase transition was evidenced, leading to a non-centrosymmetric tetragonal room temperature $14 \mathrm{~cm}$ phase. ${ }^{9}$ This phase satisfies the group-subgroup relationship along the phase transition between P4mm and $\mathrm{I} 4 \mathrm{~cm}$. We underline that a precise account of the space group is important, as the interplay between SOC and the lack of centrosymmetry leads to a splitting of the electronic bands away from the critical point. ${ }^{5}$

Next, the graphical abstract proposed by Yun Wang et al. puts forward the good agreement between the calculated DFT band gap and the experimental one, although the authors state in the manuscript "the experimental band gaps... are surprisingly close to the theoretical predictions". A similar observation, namely "quite a peculiar situation seems to exist for Pb-based perovskites, for which DFT-GGA calculated band gaps in general good agreement with experimental values have been reported. As examples, we mention that for $\mathrm{CH}_{3} \mathrm{NH}_{3} \mathrm{PbI}_{3}$ calculated band gap values in the 1.3-1.6 eV range have been found, to be compared to an experimental band gap of $1.55 \mathrm{eV}$ ", was made earlier by another group. ${ }^{10}$ Both the graphical abstract and such statements are hazardous, as it is well known that DFT is a ground state theory. ${ }^{11}$ In addition, it was clearly stated in ref. 4, and suggested in ref. 10 (cited in the work by Yun Wang et al.), that such an agreement is fortuitous. Indeed, for related compounds such as $\mathrm{CH}_{3} \mathrm{NH}_{3} \mathrm{PbBr}_{3}$ or $\mathrm{CH}_{3} \mathrm{NH}_{3} \mathrm{SnI}_{3}$, DFT band gaps (without SOC) do not agree with experimental optical band gaps. ${ }^{3-6,10}$ The numerical agreement between experiment and DFT (without SOC) stems from large error cancellations. First, the band gap is strongly reduced when taking into account SOC. ${ }^{3-6}$ This lowering is by far much larger than the small difference quoted by the authors between the PBE and the so-called "optB86b + vdWDF" exchange-correlation functionals. Next, as expected, the band gap calculated with SOC is strongly underestimated in the DFT ground-state computations. This can be cured by including many-body effects: GW self-energy corrections for the band gap and using the Bethe Salpeter equation resolution to account for excitonic effects, or by implementing TD-DFT approaches. ${ }^{4,5,11}$ This is well illustrated by the one-shot GW correction reported for the cubic phase of $\mathrm{CsPb}_{3}$, an all-inorganic analogue of $\mathrm{CH}_{3} \mathrm{NH}_{3} \mathrm{PbI}_{3}$, that amounts to a $0.6 \mathrm{eV}$ increase of the band gap at the LDA level. ${ }^{4}$ Although not self-consistent, this value indicates that GW and SOC corrections are both large and act in opposite directions. Even so, accounting both for SOC and many-body effects would need huge computational resources, the fortuitous agreement between calculated and experimental band gaps should have been discussed beyond simply quoting "hybrid" functionals.

Lastly, Bader (AIM) charges summarized in Table 3 of Yun Wang et al.'s paper are particularly striking, especially for the methylammonium cation, formally $\left(\mathrm{CH}_{3}\right) \mathrm{NH}_{3}{ }^{+}$. In fact, as stated in the manuscript "the $\mathrm{NH}_{3}$ group is almost charge neutral; and most of the positive charge is the 
contribution from the $\mathrm{CH}_{3}$ group". Contrarily to the assertion made, this is not what chemical intuition tells. Such a charge distribution may be indicative of (partial) bond breaking of the $\mathrm{C}-\mathrm{N}$ bond during crystal structure optimization. Discussion of Bader charges would have, at least, required correlation with structural information, such as the $\mathrm{C}-\mathrm{N}$ bond length and $\mathrm{HNH}$ and $\mathrm{HCH}$ bond angles, the latter being useful to check whether the $\mathrm{CH}_{3}$ moiety is still tetrahedral and not (almost) planar. It may also provide better insight into the relevance of using the "optB86b + vdWDF" exchange-correlation functional, i.e. corrected for van der Waals interactions, for this class of metal-halide perovskites that are chemically quite different from e.g. layers of carbon sheets.

\section{Acknowledgements}

C. K. would like to thank Hélène Gérard for useful discussions.

\section{Notes and references}

1. N. G. Park, J. Phys. Chem. Lett., 2013, 4, 2423

2. H. Snaith, J. Phys. Chem. Lett., 2013, 4, 3623

3. J. Even, L. Pedesseau, M.-A. Dupertuis, J.-M. Jancu and C. Katan, Phys. Rev. B: Condens. Matter Mater. Phys., 2012, 86, 205301

4. J. Even, L. Pedesseau, J.-M. Jancu and C. Katan, J. Phys. Chem. Lett., 2013, 4, 2999

5. J. Even, L. Pedesseau, J.-M. Jancu and C. Katan, Phys. Status Solidi RRL, 2014, 8, 31

6. L. Pedesseau, J.-M. Jancu, A. Rolland, E. Deleporte, C. Katan and J. Even, Opt. Quantum Electron., 2013 DOI:10.1007/s11082-013-9823-9

7. Y. Kawamura, H. Mashiyama and K. Hasebe, J. Phys. Soc. Jpn., 2002, 71, 1694

8. T. Baikie, Y. Fang, J. M. Kadro, M. Schreyer, F. X. Wei, S. G. Mhaisalkar, M. Graetzel and T. J. White, J. Mater. Chem. A, 2013, 1, 5628

9. C. C. Stoumpos, C. D. Malliakas and M. G. Kanatzidis, Inorg. Chem., 2013, 52, 9019

10. E. Mosconi, A. Amat, M. K. Nazeeruddin, M. Gratzel and F. De Angelis, J. Phys. Chem. C, 2013, 117, 13902-13913

11. G. Onida, L. Reining and A. Rubio, Rev. Mod. Phys., 2002, 74, 601 